\title{
Cancer cell line encyclopedias
}

\section{By Tim Fulmer, Senior Writer}

Two research teams have independently developed large-scale screening platforms to profile hundreds of human cancer cell lines and identify drug sensitivity biomarkers. ${ }^{1,2}$ Novartis AG, a member of one of the groups, is now using the platform to guide patient selection in Phase I cancer trials.

Both platforms are patterned after the NCI60, which was the first cancer cell line panel developed to screen for cancer therapeutics. NCI60 consists of 60 human cell lines representing 9 tumor types. It was developed in the 1980s and first used in $1990 .^{3}$

In the intervening 20 years it has become clear that the original NCI60 panel cannot capture the full diversity of cancer cell lineages and cancer genetics. ${ }^{4,5}$ The panel "is just too small to model the incredible heterogeneity that, through genome sequencing, we now know exists across cancer mutations," Paul Workman told SciBX. "To cover mutations present in, say, $5 \%$ or less of human cancers, you need to have a panel of several hundred cancer cell lines."

"To cover mutations present in, say, $5 \%$ or less of human cancers, you need to have a panel of several hundred cancer cell lines."

-Paul Workman,

The Institute of Cancer Research
Workman is deputy CEO and director of the Cancer Research UK Cancer Therapeutics Unit at The Institute of Cancer Research.

Modeling that complex heterogeneity at the molecular level is important because "it helps us identify biomarkers of drug sensitivity and drug resistance that lead to personalized cancer treatments," said Ultan McDermott, corresponding author on one of the papers and group leader of the Wellcome Trust Sanger Institute's Cancer Genome Project.

Thus, the first priority was to assemble a large set of cancer cell lines that might reflect the molecular and genetic heterogeneity of tumor tissues.

The Wellcome-Massachusetts General Hospital group, led by Andrew Futreal, Michael Stratton and Daniel Haber, collected 639 human tumor cell lines representing a range of adult and childhood cancers of epithelial, mesenchymal and hematopoietic origin. Lung, blood, CNS, GI tract, skin and breast cancers were the most highly represented in the panel.

Futreal is head of cancer genetics at Wellcome, and Stratton is director of Wellcome. Futreal and Stratton also are joint heads of Wellcome's Cancer Genome Project. Haber is professor of biological and biomedical sciences at Harvard Medical School and director of the Massachusetts General Hospital Cancer Center.

The Novartis-Broad Institute of MIT and Harvard group, led by Robert Schlegel, William Sellers and Levi Garraway, assembled a panel of 947 human cancer cell lines dubbed the Cancer Cell Line Encyclopedia (CCLE), which encompassed 36 tumor types. The most highly represented cancers in that panel were lung, colorectal, melanoma, breast, ovary, glioma and pancreatic.

Schlegel is executive director of oncology at Novartis. Sellers is VP and global head of oncology at Novartis. Garraway is a researcher at the Broad Institute, professor of biological chemistry and molecular pharmacology at Harvard Medical School and assistant professor of medicine at the Dana-Farber Cancer Institute.

For both groups, the number of cell lines per cancer was broadly a function of the mortality rate of a given cancer and the availability of cell lines for that cancer.

The next step for the teams was generating a set of molecular-level biomarkers. To do so, the groups characterized each cell line according to several basic genetic traits. The Wellcome-MGH group used standard capillary sequencing to measure the mutational status of 64 well-known cancer-associated genes. In addition, they measured DNA copy number and the expression of 14,500 genes.

The Novartis-Broad group used massively parallel sequencing to determine the mutational status of 1,600 cancer-associated genes, encompassing well-known cancer genes as well as genes identified in the literature and presentations as being putative oncogenes and tumor suppressors. In addition, the group measured DNA copy number and genome-wide mRNA expression.

With cancer cell lines and corresponding biomarkers in hand, the groups each screened a compound library to identify drug-sensitive and drug-resistant cell lines.

The Wellcome-MGH group selected 130 compounds, including targeted molecules and chemotherapeutics, and assayed 48,178 compound-cell line combinations, with a mean of 368 cell lines screened per compound.

For each compound-cell line combination, the effect of 72 hours of treatment was used to derive a drug sensitivity profile that incorporated the $\mathrm{IC}_{50}$ value and a dose-response curve. The researchers then correlated drug sensitivity and genetic traits, generating genecompound associations that predicted sensitivity and resistance.

The screen identified highly significant correlations between known oncogenes and drugs. For example, the $B C R-A B L$ gene rearrangement predicted sensitivity to BCR-ABL tyrosine kinase inhibition $\left(p=2.54 \times 10^{-65}\right)$; $B R A F$ mutational status predicted sensitivity to BRAF inhibition $\left(p=1.25 \times 10^{-24}\right)$; HER2 (EGFR2; ERBB2; neu) amplification predicted sensitivity to epidermal growth factor receptor (EGFR) inhibition $\left(p<1 \times 10^{-7}\right) ; p 53$ mutational status predicted resistance to $\mathrm{mdm} 2 \mathrm{p} 53$ binding protein homolog (MDM2; HDM2) inhibition $\left(p<1 \times 10^{-36}\right)$.

As proof the platform could identify new biomarkers of drug sensitivity, the researchers found that the Ewing sarcoma breakpoint region 1 (EWSR1; EWS)-Friend leukemia virus integration 1 (FLI1) gene rearrangement predicted a previously unknown sensitivity of Ewing's 
sarcoma cells to poly(ADP-ribose) polymerase (PARP) inhibitors $\left(p=1.03 \times 10^{-26}\right)$. Subsequent cell culture studies confirmed PARP inhibition induced apoptosis in Ewing's sarcoma cell lines after 72 hours of treatment.

The Novartis-Broad group selected 24 compounds, both targeted therapies and chemotherapeutics, and screened them in 479 cell lines, generating drug sensitivity profiles for each compound-cell line combination.

Statistical analysis of those results identified many of the same known biomarkers of drug sensitivity found by the Wellcome-Harvard team's platform. The Novartis-Broad group also identified previously unknown sensitivity biomarkers: elevated expression of the aryl hydrocarbon receptor (AHR) gene in neuroblastoma Ras viral oncogene (NRAS)mutant cells predicted sensitivity to MEK inhibition, and elevated expression of the schlafen family member 11 (SLFN11) gene predicted sensitivity to topoisomerase inhibitors in multiple cell lineages. Both of those predictions were subsequently confirmed in cell culture studies.

The Wellcome-Harvard findings and the Novartis-Broad findings were published in Nature.

\section{Early and late}

The screening platforms may turn out to be useful both early and late in the preclinical drug development process. "Early on, these large, unbiased screens provide insight into how certain mutations drive drug sensitivity and resistance at the molecular level. In other words, they allow us to match drugs to mutations," said McDermott. "However, to account for the effects of tumor biology, angiogenesis and stroma on a drug, these screens will have to be supplemented with more complex animal models down the line."

Later in preclinical development, following animal testing, the drug sensitivity and resistance profiles could again become useful, said McDermott. "In this case, the biomarkers of drug sensitivity identified in our cell lines are now used to generate genetic profiles of patients and thus help inform trial design and patient stratification."

Novartis is already using its screening platform to help guide patient selection in its Phase I cancer trials, said Sellers. "For example, a trial for the PI3K [phosphoinositide 3-kinase] inhibitor BYL719 was focused on patients who show specific mutations in the PI3KCA gene in part motivated by the strength of the cancer cell line encyclopedia data." BYL719 is in Phase I testing to treat solid tumors.

Moving forward, both groups plan to build out their screening platforms with additional biomarkers and drug combinations.

"We plan to characterize our cell line panel at the level of epigenetics, looking at patterns of histone modifications and DNA methylation as potential biomarkers of drug sensitivity," said McDermott. "We also want to assay combinations of drugs in our cell lines. Finally, we want to optimize and unify our analytical methods into a single statistical algorithm."

The Novartis-Broad group "wants to include a variety of additional molecular-level biomarker profiles, including epigenetics, metabolomics, proteomics and microRNA expression levels. We also want to supplement genome sequencing with RNA sequencing," said Sellers.

Novartis also is developing a human xenograft mouse tumor encyclopedia that will supplement the cell line screen, said Sellers. "The mouse encyclopedia will encompass a wide variety of tumor types in an in vivo setting. It will help provide insight into how tumor stromaepithelial interactions affect drug activity, which is impossible to assay in a cell line platform."

The findings in both papers are unpatented and are freely available to the public at http://www.broadinstitute.org/ccle and http://www.cancerrxgene.org/.

Fulmer, T. SciBX 5(17); doi:10.1038/scibx.2012.431

Published online April 26, 2012

\section{REFERENCES}

1. Barretina, J. et al. Nature; published online March 28, 2012; doi:10.1038/nature11003

Contact: Levi A. Garraway, Dana-Farber Cancer Institute, Boston, Mass.

e-mail: levi_garraway@dfci.harvard.edu

Contact: Robert Schlegel, Novartis Institutes for BioMedical Research, Cambridge, Mass.

e-mail: robert.schlegel@novartis.com

2. Garnett, M.J. et al. Nature; published online March 28, 2012; doi:10.1038/nature11005

Contact: Ultan McDermott, Wellcome Trust Sanger Institute, Hinxton, U.K.

e-mail: um1@sanger.ac.uk

Contact: Cyril H. Benes, Harvard Medical School, Boston, Mass. e-mail: cbenes@partners.org

3. Shoemaker, R.H. Nat. Rev. Cancer 6, 813-823 (2006)

4. Caponigro, G. \& Sellers, W.R. Nat. Rev. Drug Discov. 10, 179-187 (2011)

5. Workman, P. et al. Cancer Cell 21, 455-458 (2012)

COMPANIES AND INSTITUTIONS MENTIONED

Broad Institute of MIT and Harvard, Cambridge, Mass.

Cancer Research UK, London, U.K.

Dana-Farber Cancer Institute, Boston, Mass.

Harvard Medical School, Boston, Mass.

The Institute of Cancer Research, London, U.K.

Massachusetts General Hospital, Boston, Mass.

Massachusetts General Hospital Cancer Center, Boston, Mass.

Novartis AG (NYSE:NVS; SIX:NOVN), Basel, Switzerland

Wellcome Trust Sanger Institute, Hinxton, U.K. 